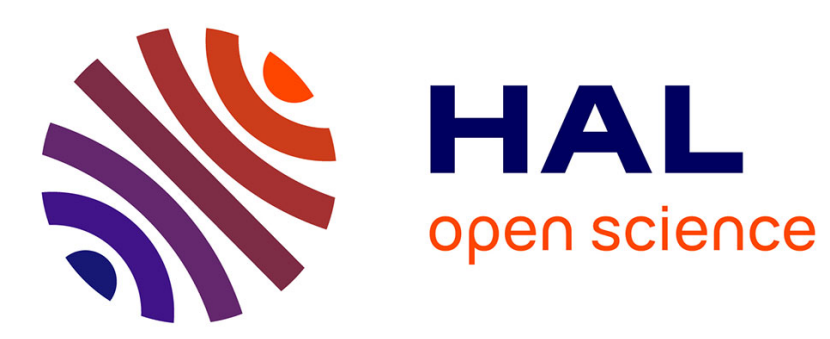

\title{
Influence des dimensions de grains sur l'anomalie de la résistivité du nickel autour du point de Curie
}

Th. Rappeneau

\section{To cite this version:}

Th. Rappeneau. Influence des dimensions de grains sur l'anomalie de la résistivité du nickel autour du point de Curie. Journal de Physique Lettres, 1976, 37 (9), pp.223-227. 10.1051/jphyslet:01976003709022300 . jpa-00231279

\section{HAL Id: jpa-00231279 https://hal.science/jpa-00231279}

Submitted on 1 Jan 1976

HAL is a multi-disciplinary open access archive for the deposit and dissemination of scientific research documents, whether they are published or not. The documents may come from teaching and research institutions in France or abroad, or from public or private research centers.
L'archive ouverte pluridisciplinaire HAL, est destinée au dépôt et à la diffusion de documents scientifiques de niveau recherche, publiés ou non, émanant des établissements d'enseignement et de recherche français ou étrangers, des laboratoires publics ou privés. 


\title{
INFLUENCE DES DIMENSIONS DE GRAINS SUR L'ANOMALIE DE LA RÉSISTIVITÉ DU NICKEL AUTOUR DU POINT DE CURIE
}

\author{
Th. RAPPENEAU \\ Laboratoire d'Optique des Solides, \\ Université Pierre et Marie Curie, 75230 Paris Cedex 05, France
}

(Reçu le 22 mars 1976, révisé le 11 juin 1976, accepté le 14 juin 1976)

\begin{abstract}
Résumé. - Les mesures de résistivité effectuées sur du nickel formé de cristaux très petits (15 $\AA$, $25 \AA$ ) ont permis de vérifier les résultats théoriques concernant l'abaissement de la température de Curie, l'arrondissement de la courbe $\alpha(T)=\mathrm{d} \rho / \mathrm{d} T$ et de calculer l'exposant critique $v$ et la constante $\xi_{0}$ liés à la longueur de corrélation.

Les valeurs du libre parcours moyen $l$ des électrons et la variation de $\rho_{\mathrm{i}}(T)$ (résistivité due aux imperfections du réseau et joints de grains) sont interprétées qualitativement en liaison avec les fluctuations de spins et les tailles des cristaux.
\end{abstract}

\begin{abstract}
A fractional shift in critical temperature and a rounding of the $\alpha(T)=\mathrm{d} \rho / \mathrm{d} T$ peak are observed on very small grain-sized nickel (15 $\AA, 25 \AA)$. The critical exponent $v$ and the constant $\xi_{0}$ are computed. Results are compared with theoretical data.

The behaviour of the electron mean free path $l$ and of $\rho_{i}(T)$ (resistivity term due to defects and grain boundaries) is qualitatively related to spin fluctuations and grain size.
\end{abstract}

1. Introduction. - Les études théoriques des phénomènes critiques $[1,2,3]$ sont généralement effectuées sur des modèles infinis et homogènes. Assez récemment, Fisher et Ferdinand [4,5] prirent en considération le fait que les systèmes physiques réels étaient finis et possédaient des limites (surfaces et interfaces, joints de grains) ainsi que des défauts (impuretés, défauts ponctuels, dislocations, tensions). Aussi ontils repris leurs calculs sur la base de modèles présentant des limites et représentatifs de l'existence des joints de grains. Leurs résultats font apparaître que les dimensions des cristaux sont responsables d'un déplacement du point critique et d'un arrondissement des pics caractéristiques des anomalies au voisinage des points critiques. Ces phénomènes sont difficilement décelables. Ils ont néanmoins été observés au cours de mesures très précises sur des ferromagnétiques $[6,7,8,9]$. Leur étude serait plus aisée sur des matériaux présentant des dimensions de grains très inférieures à celles que l'on rencontre normalement dans les échantillons massifs, mais qu'il est possible d'obtenir sur des couches minces préparées par évaporation sous vide.

L'objet de cette lettre est de présenter les résultats d'un travail expérimental sur l'anomalie de la résistivité de couches minces de nickel à structure microcristalline dans lesquelles l'épaisseur des couches joue un rôle négligeable devant les dimensions des cristaux et de les comparer aux résultats théoriques relatifs aux phénomènes critiques.

\section{Méthodes de mesures et résultats expérimentaux.} - Les couches ont été déposées sur des supports en silice ou en verre par évaporation thermique, sous ultra-vide, de nickel de pureté 0,99998 et stabilisées in situ par un recuit de plusieurs heures entre $400{ }^{\circ} \mathrm{C}$ et $500^{\circ} \mathrm{C}$. Les mesures de résistance furent ensuite effectuées également in situ.

Les renseignements sur la structure cristallographique de ces couches ont été fournis par étude au microscope électronique de répliques de carbone effectuées après attaque chimique des joints de grains. Nous nous intéresserons plus particulièrement à deux types de structure :

a) homogène : cristaux de même taille (environ $15 \AA$ ) pour une couche d'épaisseur $95 \AA$;

b) hétérogène : cristaux de tailles diverses $(25 \AA$, 30 à $50 \AA$ et quelques cristaux plus gros de $100 \AA$ à $150 \AA$ ) pour une couche d'épaisseur $510 \AA$ évaporée en trois fois, les recuits intermédiaires ayant provoqué l'agglomération des premiers dépôts. Les épaisseurs ont été mesurées aux rayons $\mathrm{X}$ par la méthode de Kiessig [10]. 
A partir des mesures de la résistance et des dimensions des couches, nous avons pu déterminer leur résistivité et en déduire, à l'aide de la théorie de Fuchs [11] sur les effets de dimension dans les couches minces, la résistivité $\rho$ qu'elles présenteraient pour une épaisseur infinie. $\rho$ est donc caractéristique de la structure cristallographique du nickel des couches. Les valeurs du libre parcours moyen des électrons de conduction $l$ sont déduites de $\rho$ sachant que le produit $\rho . l$ est une constante caractéristique de métal [12]. La figure 1 montre les courbes $\rho(T)$ relatives aux deux types d'échantillons de nickel obtenus ainsi que $\rho(T)$ pour un échantillon de nickel massif [13].

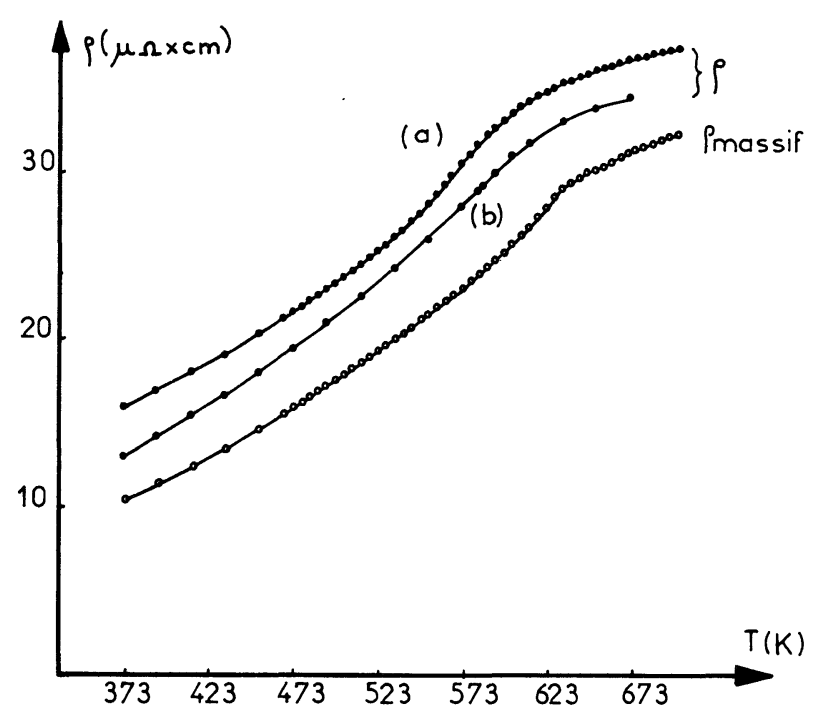

FIG. 1. - Variation de la résistivité en fonction de la température ; (a) $\rho(T)$ pour l'échantillon à structure microcristalline homogène à grains très fins. $(b) \cdot \rho(T)$ pour l'échantillon à structure inhomogène. La courbe $\rho(T)$ pour le nickel massif a été tracée d'après les valeurs fournies par Potter [13].

Les températures de Curie correspondantes ont été déterminées à partir du maximum des courbes

$$
\alpha(T)=\frac{\mathrm{d} \rho(T)}{\mathrm{d} T} \quad(\text { Fig. 2) }
$$

Elles sont inférieures de plusieurs dizaines de degrés au point de Curie du nickel massif et nous les noterons $T_{\mathrm{c}}^{\prime}$.

La résistivité $\rho(T)$ peut être décomposée en deux termes : $\rho_{\mathrm{e}}(T)$ dû aux interactions électrons de conduction/phonons ainsi qu'aux interactions d'origine magnétique pour les ferromagnétiques [14] et $\rho_{\mathrm{i}}(T)$ dû à la diffusion des électrons par les imperfections du réseau (joints de grains et défauts). $\rho_{\mathrm{i}}$ est très petit devant $\rho_{\mathrm{e}}$ dans le cas du nickel massif, mais ici $\rho_{\mathrm{i}}$ calculé à partir de la relation : $\rho_{\mathrm{i}}(T)=\rho(T)-\rho_{\mathrm{e}}(T)$ est du même ordre de grandeur que $\rho$ (environ $\rho / 5$ ) et augmente lorsque la taille des cristaux diminue.

3. Loi de divergence. - La loi de divergence caractéristique des anomalies de la résistivité et de la chaleur spécifique des ferromagnétiques peut s'écrire [15],
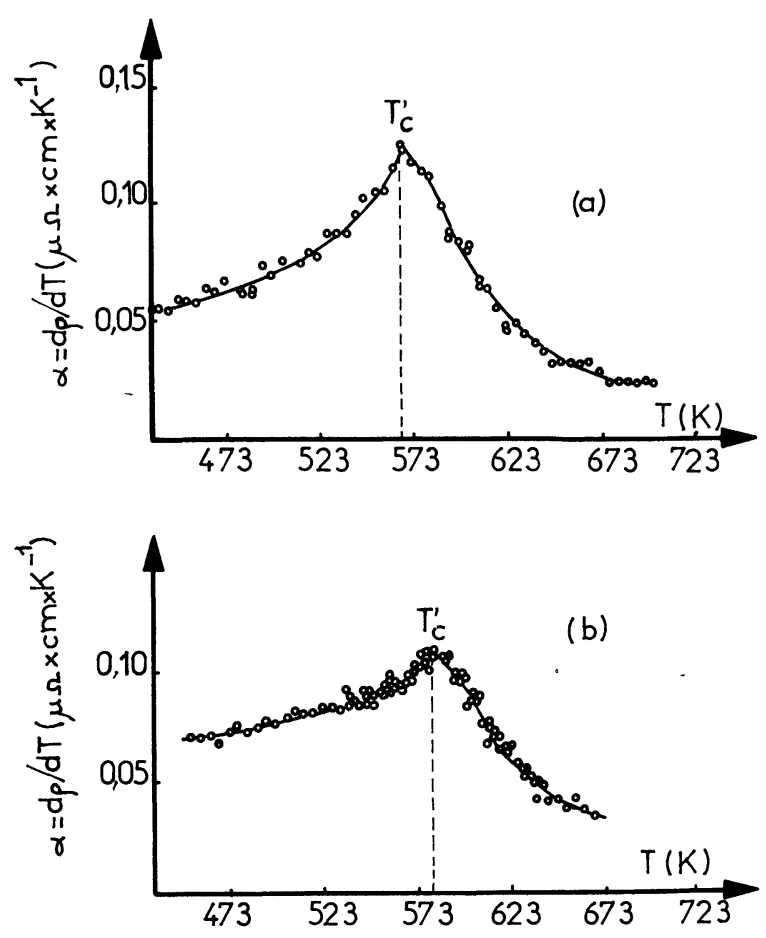

Fig. 2. - Variation de $\alpha=\mathrm{d} \rho / \mathrm{d} T$ en fonction de la température ; (a) échantillon à structure homogène très fine : $T_{\mathrm{c}}^{\prime}=567 \mathrm{~K}$; (b) échantillon à structure inhomogène fine : $T_{\mathrm{c}}^{\prime}=582 \mathrm{~K}$.

dans le cas d'un échantillon inhomogène et de dimensions finies présentant un point de Curie $T_{\mathrm{c}}^{\prime}$ diffèrent du point $T_{\mathrm{c}}$ caractéristique du métal idéal (homogène et infini), sous la forme :

$$
\left(\frac{\mathrm{d} \alpha}{\mathrm{d} T}\right)_{+}=-A_{+}\left(\frac{\left|T_{\mathrm{c}}^{\prime}-T\right|}{T_{\mathrm{c}}}\right)^{-\left(\lambda_{+}+1\right)}
$$

pour les températures supérieures à $T_{\mathrm{c}}^{\prime}$ et

$$
\left(\frac{\mathrm{d} \alpha}{\mathrm{d} T}\right)_{-}=-A_{-}\left(\frac{\left|T_{\mathrm{c}}^{\prime}-T\right|}{T_{\mathrm{c}}}\right)^{-\left(\lambda_{-}+1\right)}
$$

pour les températures inférieures à $T_{\mathrm{c}}^{\prime}$.

$A_{+}$et $A_{-}$sont des constantes et $\lambda_{+}, \lambda_{-}$caractérisent la divergence de $\alpha$. Nous avons effectivement vérifié cette loi (Fig. 3 et 4 ) avec les valeurs suivantes des coefficients $\lambda$ :

échantillon $a)$ :

$$
\lambda_{+}=+0,11 \pm 0,05 ; \quad \lambda_{-}=-0,39 \pm 0,05
$$

échantillon $b$ ) :

$$
\lambda_{+}=+0,05 \pm 0,05 ; \quad \lambda_{-}=-0,28 \pm 0,02 .
$$

Ces valeurs sont en accord avec les résultats obtenus sur du nickel massif $[6,7,8,16,17]: \lambda_{+}=0$ (divergence logarithmique); $\lambda_{-} \approx-0,3$ et plus rarement $\lambda_{-}=0$. Théoriquement $[2,18], \lambda_{-}$est de l'ordre de $-0,375$. 

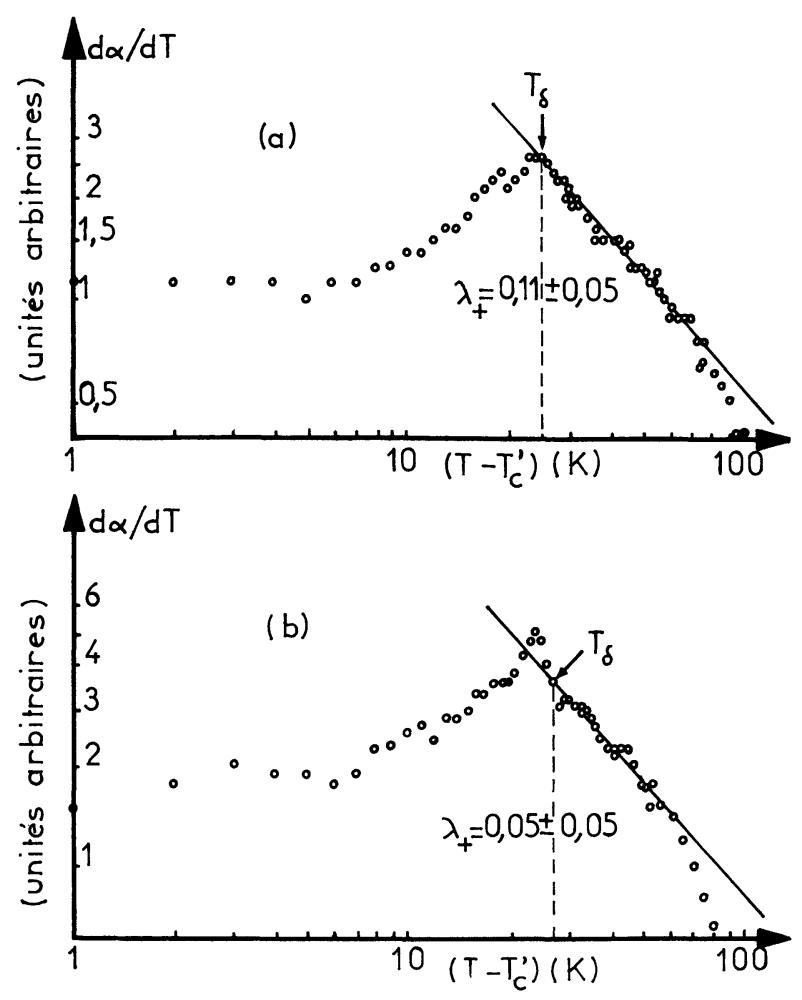

Fig. 3. - Variation de $\mathrm{d} \alpha / \mathrm{d} T$ en fonction de $T-T_{\mathrm{c}}^{\prime}$ au-dessus du point $T_{\mathrm{c}}^{\prime} ;(a)$ échantillon à structure homogène très fine $: T_{\delta}=592 \mathrm{~K}$; (b) échantillon à structure inhomogène fine : $T_{\delta}=609 \mathrm{~K}$.
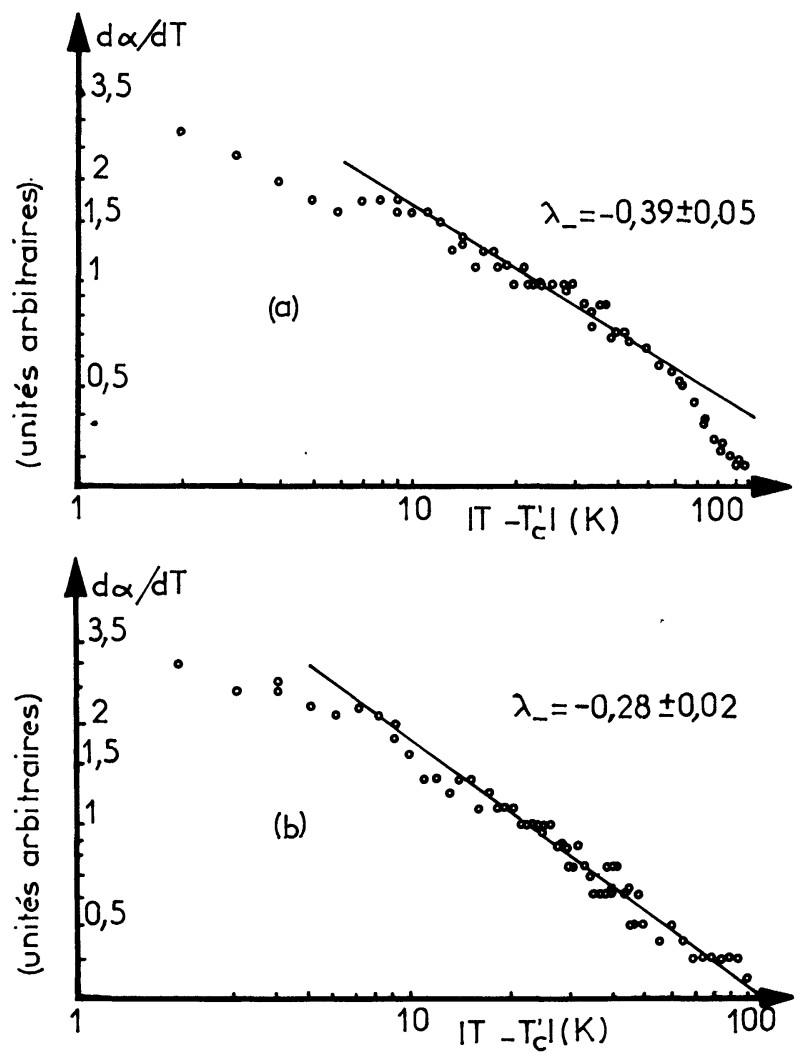

Fig. 4. - Variation de $\mathrm{d} \alpha / \mathrm{d} T$ en fonction de $\left|T-I_{\mathrm{c}}^{\prime}\right|$ au-dessous du point $T_{\mathrm{c}}^{\prime} ;(a)$ échantillon à structure homogène très fine; $(b)$ échantillon à structure inhomogène fine.
4. Abaissement relatif de la température de Curie. Expérimentalement, nous avons trouvé que le déplacement relatif du point du Curie,

$$
\varepsilon=\frac{T_{\mathrm{c}}-T_{\mathrm{c}}^{\prime}}{T_{\mathrm{c}}}
$$

était proportionnel au rapport $\rho_{\mathrm{i}} / \rho$ calculé à la température $T_{\mathrm{c}}^{\prime}$ :

$$
\varepsilon=0,43\left(\frac{\rho_{\mathrm{i}}}{\rho}\right)_{T_{\mathrm{c}}^{\prime}}
$$

$T_{\mathrm{c}}$ étant égal, à une fraction de degré près, à la température de Curie du nickel massif très pur, nous l'avons pris égal à $630 \mathrm{~K}$, valeur observée sur des échantillons de nickel de grande pureté [13]. Les températures de Curie $T_{\mathrm{c}}^{\prime}$, déterminées à partir des courbes $\alpha(T)$, sont $567 \mathrm{~K}$ et $582 \mathrm{~K}$ respectivement pour les échantillons $a$ ) et $b$ ).

5. Arrondissement de $\alpha(T)$; exposant critique; longueur de corrélation. - On définit une température $T_{\delta}$ telle que, pour $T \geqslant T_{\delta}, \mathrm{d} \alpha / \mathrm{d} T$ est exactement représenté par l'équation (1). $T_{\delta}$ est déterminé sur les courbes de la figure 3 . Si l'on définit un arrondissement relatif

$$
\Delta_{\exp }=\frac{T_{\mathrm{c}}-T_{\delta}}{T_{\mathrm{c}}}
$$

on trouve que $\Delta_{\exp }$ est lié au rapport $\left(\rho_{\mathrm{i}} / \rho\right)$ calculé à la température $T_{\delta}$ suivant la loi :

$$
\Delta_{\exp }=0,49\left(\frac{\rho_{\mathrm{i}}}{\rho}\right)_{T_{\delta}}^{1 / 0,68} .
$$

Fisher et Ferdinand $[4,5]$ trouvent que l'arrondissement relatif, lié à la taille moyenne $\bar{n} a$ des cristaux du système supposé homogène ( $a=$ constante du réseau), a pour expression $c / \bar{n}^{1 / v}$ où $c$ est une constante caractéristique du système et $v$ l'exposant critique lié à la longueur de corrélation :

$$
\xi=\xi_{0}\left(\frac{T_{\mathrm{c}}-T}{T_{\mathrm{c}}}\right)^{-v}
$$

$v$ apparaît ici car, à la température $T_{\delta}$, la longueur $\bar{n} a$ caractéristique des dimensions du système réel doit être voisine de la longueur de corrélation du système idéal. Ceci revient à écrire que :

$$
\Delta_{\text {théorique }}=\frac{\left|T_{\mathrm{c}}-T_{\delta}\right|}{T_{\mathrm{c}}}=\frac{c}{\bar{n}^{1 / v}} .
$$

Or, l'exposant 0,68 trouvé expérimentalement correspond à la valeur de $v$ la plus couramment admise, $\approx \frac{2}{3}$.

D'autre part, $\left(\rho / \rho_{\mathrm{i}}\right)_{T_{\delta}} . a$ se trouve égal à la dimension moyenne $\bar{n} a$ des cristaux (15 ̊ pour l'échantillon à structure microcristalline homogène très fine, snit 
la taille observée pour les grains; 22 A pour l'échantillon à structure microcristalline inhomogène, valeur légèrement inférieure à la taille latérale des plus petits grains $(25 \AA)$ mais compatible avec une épaisseur des cristaux légèrement inférieure à leurs dimensions dans le plan de la couche). Il apparaît ainsi que $\Delta_{\text {th }}=\Delta_{\text {exp }}$ et que $\rho_{\mathrm{i}} / \rho$ caractérise essentiellement la résistivité due aux joints de grains. Enfin, $\left(\rho / \rho_{\mathrm{i}}\right) \cdot a$ doit être égal à $\xi_{T_{\delta}}$, ce qui d'après la formule (2) donne $\xi_{0}=2 \AA$. C'est la valeur proposée par Fisher [19] pour $\xi_{0}$.

6. Libre parcours moyen des électrons de conduction. - Les résultats précédents concernent les phénomènes critiques en général, quelle qu'en soit la nature. Il reste à étudier l'influence de la structure cristallo-. graphique sur le comportement des électrons de conduction, caractérisé par le libre parcours moyen $l$.

Fisher et Langer [18] estiment que à $-10 \mathrm{~K}$ de $T_{\mathrm{c}}$, $l$ est de l'ordre de grandeur de $\xi$ dans le cas du nickel massif. La figure 5 représente les courbes $\xi(T)$ et $l(T)$ déduites des résultats précédents ainsi que $l(T)$ obtenu sur un échantillon de nickel massif. Leurs intersections se situent à $T_{\mathrm{c}}-11 \mathrm{~K}$ (nickel massif);

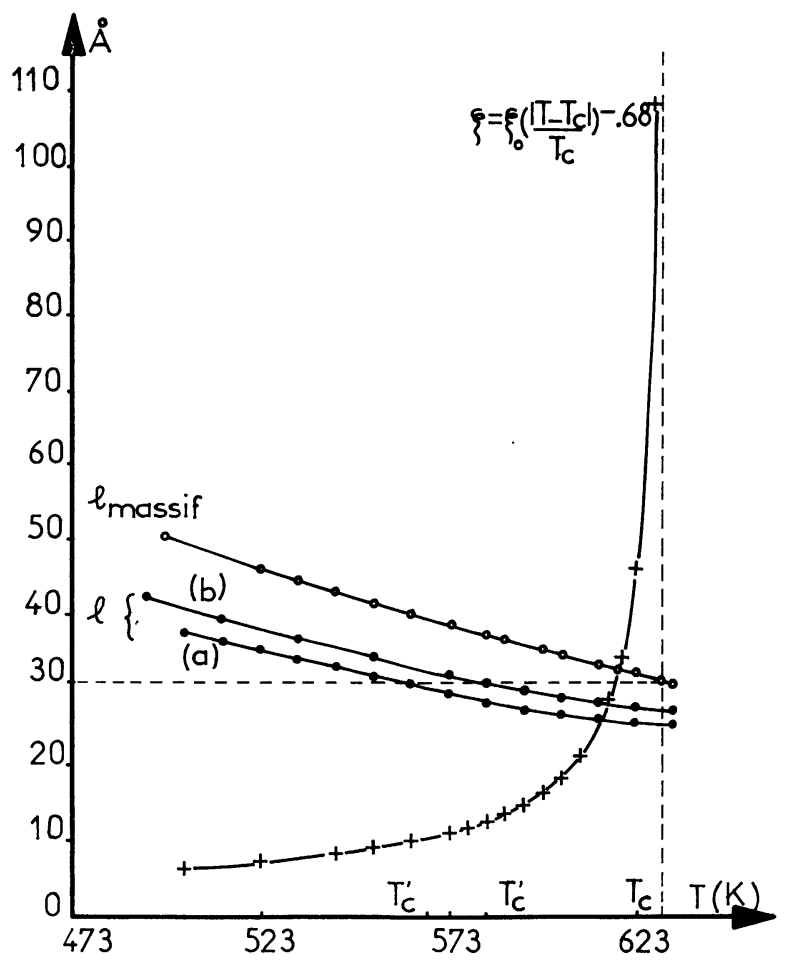

FIG. 5. - Courbe

$$
\xi(T)=\xi_{0}\left(\frac{\left|T-T_{\mathrm{c}}\right|}{T_{\mathrm{c}}}\right)^{-v}
$$

tracée avec les valeurs $\xi_{0}=2 \AA$ et $v=0,68$ calculées à partir de nos mesures. Les valeurs de $l$ et $l_{\text {massif }}$ sont déduites de $\rho$ et $\rho_{\text {massif }}$ à partir de la relation :

$$
\rho . l=\left(\frac{3}{8 \pi}\right)^{1 / 3} \frac{h}{\mathrm{e}^{2}} n^{-2 / 3}=8,90 \times 10^{-12} \Omega . \mathrm{cm}^{2} .
$$

(a) Echantillon à structure homogène très fine ; $(b)$ Echantillon à structure inhomogène fine.
$\Gamma_{\mathrm{c}}-15 \mathrm{~K}$ (grains fins) et $T_{\mathrm{c}}-16 \mathrm{~K}$ (grains très fins). L'écart augmente lorsque les cristaux deviennent plus petits par suite de la diminution du libre parcours moyen.

Les mêmes auteurs [18] attribuent l'augmentation monotone de la résistivité du nickel autour du point de Curie à un amortissement de la fonction de corrélation spin-spin $\Gamma(\mathbf{R})$ par un facteur $p(\mathbf{R})$ de la forme $\exp (-R / l)$ où $R$ est la distance entre spins. Or on constate que $l$ a sensiblement la même valeur pour nos deux échantillons que pour le nickel massif à leurs températures de Curie respectives (Fig. 5). On peut donc admettre que l'arrondissement des courbes $\rho(T)$ et $\alpha(T)$ dans le cas d'échantillons à grains très fins, de dimension $\bar{n} a$ inférieure à $l$, correspond à un amortissement plus important de la fonction $\Gamma(\mathbf{R})$.

7. Diffusion critique des électrons au voisinage de $\boldsymbol{T}_{\mathrm{c}}^{\prime}$. - L'augmentation importante de $\rho_{\mathrm{i}}$ au voisinage de la température $T_{\mathrm{c}}^{\prime}$ peut être attribuée à la diffusion des électrons de conduction due aux fluctuations de spins. Les courbes $\rho_{\mathrm{i}}(T)$ (Fig. 6) rappellent en effet l'aspect des courbes représentant la transformée de Fourier $\hat{\Gamma}(\mathbf{K}, T)$ de la fonction de corrélation de densité de spin pour différentes valeurs d'un vecteur d'onde effectif $K$ lié au réseau [18]. Le maximum de $\rho_{\mathrm{i}}(T)$ se situe à une température $T_{\mathrm{MAX}}$ supérieure à $T_{\mathrm{c}}^{\prime}$ et on constate que $\left(T_{\mathrm{MAX}}-T_{\mathrm{c}}^{\prime}\right)$ augmente lorsque les dimensions des grains diminuent $(16 \mathrm{~K}$ pour l'échantillon $b$ ) et $26 \mathrm{~K}$ pour l'échantillon $a$ )).

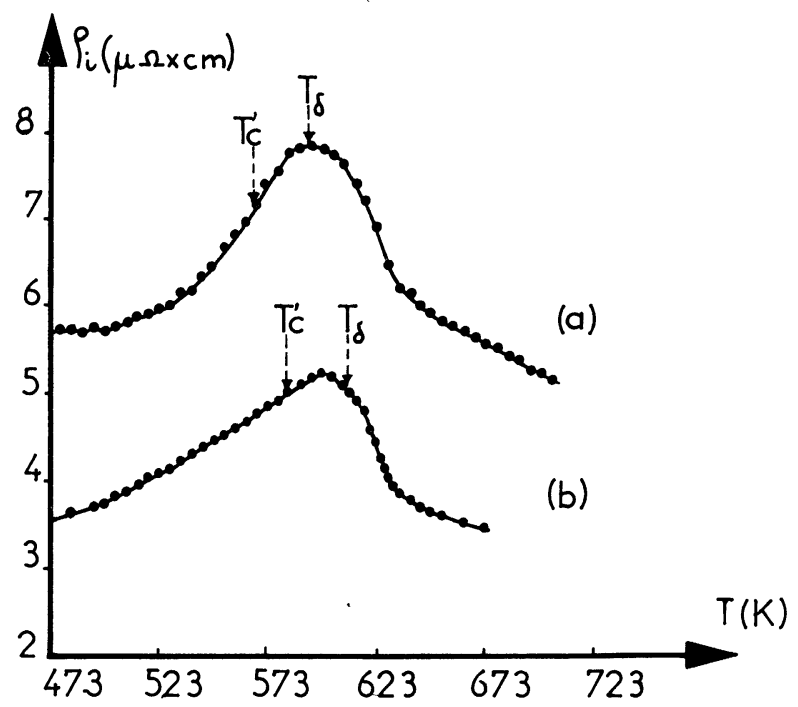

FIG. 6. - Courbes $\rho_{\mathrm{i}}(T):(a)$ Echantillon à structure homogène très fine; $(b)$ Echantillon à structure inhomogène fine.

En outre, la structure joue un rôle important dans l'allure des courbes. A une structure cristallographique homogène correspond une coụrbe caractéristique de $\hat{\Gamma}(\mathrm{K}, T)$ avec $T_{\mathrm{MAX}}=T_{\delta}$. On retrouve bien le fait qu'à $T_{\mathrm{MAX}}, \xi$ est égal à la dimension moyenne des grains $\bar{n} a$ (l'inverse de $\bar{n} a$ représentant $K$ ). Par contre, dans le cas de l'échantillon inhomogène, $T_{\mathrm{MAX}}$ est différent de $T_{\delta}$ : la courbe se présente comme 
l'enveloppe de courbes relatives aux différentes tailles de grains et $T_{\text {MAx }}$ est inférieur à $T_{\delta}$ en raison de la contribution de cristaux plus gros que ceux qui intervenaient pour fixer la valeur $T_{\delta}$.

8. Conclusions. - L'étude de la résistivité, dans le domaine critique, d'échantillons de nickel à structure microcristalline a permis de vérifier les résultats théoriques concernant un modèle de dimensions finies et de calculer les coefficients et exposants cri- tiques $\lambda, v$ et $\xi_{0}$. La diffusion critique des électrons de conduction par les fluctuations de spins se manifeste dans le terme de résistivité dû aux joints de grains. L'amortissement de la fonction de corrélation spinspin paraît dû à la limitation de la distance d'interaction entre spins par les joints de grains.

L'ensemble du travail dont cette lettre présente les résultats fera l'objet d'une publication plus complète et plus explicite.

\section{Bibliographie}

[1] De Gennes, P. G., Friedel, J., J. Phys. \& Chem. Solids 4 (1958) 71.

[2] Fisher, M. E., J. Math. Phys. 5 (1964) 944.

[3] Fisher, M. E., BURford, R. J., Phys. Rev. 156 (1967) 583.

[4] Fisher, M. E., Ferdinand, A. E., Phys. Rev. Lett. 19 (1967) 169.

[5] Ferdinand, A. E., Fisher, M. E., Phys. Rev. 185 (1969) 832.

[6] Craig, P. P. et al., Phys. Rev. Lett. 19 (1967) 1334.

[7] Craig, P. P., Goldburg, W. I., J. Appl. Phys. 40 (1969) 964.

[8] Nagy, I., PaL, L., Phys. Rev. Lett. 24 (1970) 894.

[9] Cadieu, F. J., Douglass, D. H., Phys. Rev. Lett. 21 (1968) 680.
[10] Umrath, W., Z. Angew. Phys. 22 (1967) 406

[11] SONdheimer, E. H., Adv. Phys. 1 (1952) 1.

[12] Broquet, P., NGuYen Van, V., Surf. Sci. 6 (1967) 98.

[13] Potter, H. H., Proc. Phys. Soc. 49 (1937) 671.

[14] Motr, N. F., Proc. R. Soc. A 153 (1936) 699.

[15] Fisher, M. E., Barber, M. N., Phys. Rev. Lett. 28 (1972) 1516.

[16] Zumsteg, F. C., Parks, R. D., Phys. Rev. Lett. 24 (1970) 520.

[17] Maher, W. E., McCormick, W. D., Phys. Rev. 183 (1969) 573.

[18] Fisher, M. E., LANGer, J. S., Phys. Rev. Lett. 20 (1968) 665.

[19] Langer, J. S., Fisher, M. E., Phys. Rev. Lett. 19 (1967) 560. 